\title{
Vectorial Solution to Double Curl Equation With Generalized Coulomb Gauge for Magnetostatic Problems
}

\author{
Yan-Lin $\mathrm{Li}^{1}$, Sheng $\mathrm{Sun}^{1}$, Qi I. Dai ${ }^{2}$, and Weng Cho Chew ${ }^{2}$, Fellow, IEEE \\ ${ }^{1}$ Department of Electrical and Electronic Engineering, The University of Hong Kong, Hong Kong \\ ${ }^{2}$ Department of Electrical and Computer Engineering, University of Illinois at Urbana-Champaign, \\ Champaign, IL 61801 USA
}

\begin{abstract}
In this paper, a solution to the double curl equation with generalized Coulomb gauge is proposed based on the vectorial representation of the magnetic vector potential. Traditional Coulomb gauge is applied to remove the null space of the curl operator and hence the uniqueness of the solution is guaranteed. However, as the divergence operator cannot act on edge elements (curl-conforming) directly, the magnetic vector potential is represented by nodal elements, which is too restrictive, since both the tangential continuity and the normal continuity are required. Inspired by the mapping of Whitney forms by mathematical operators and Hodge (star) operators, the divergence of the magnetic vector potential, as a whole, can be approximated by Whitney elements. Hence, the magnetic vector potential can be expanded by the edge elements, where its vectorial nature is retained and only the tangential continuity is required. Finally, the original equation can be rewritten in a generalized form and solved in a more natural and accurate way using finite-element method.
\end{abstract}

Index Terms-Finite-element method (FEM), generalized Coulomb gauge, magnetostatic, Whitney forms.

\section{INTRODUCTION}

A S A STATIC limit of Maxwell's equations, magnetostatics has many applications in Hall-effect devices, toroidal coils, magnetic resonance imaging, and so on [1]-[3]. For some simple structures with given current flow, such as air core inductors and transformers, the magnetic field induced by the current density can be analytically computed by the Biot-Savart law. However, numerical methods are inevitably used to model complex structures, especially inhomogeneous and anisotropic ones. Over the past decades, several formulations based on scalar and vector potentials, or field and flux [4]-[8] have been proposed to solve the magnetostatic problems, where the unknowns of the system of equations might be different. Among these formulations, the double curl equation pertinent to the magnetic vector potential $\mathbf{A}$ is commonly adopted in both commercial and free softwares, e.g., ANSYS Maxwell [9] and finite-element method (FEM) magnetics [10].

It is well known that the curl operator has null space (gradient of any scalar field), which makes the solution of $\mathbf{A}$ nonunique. For many applications, the magnetic flux $\mathbf{B}$ is the primary quantity of interest. After recovering $\mathbf{B}$ by the relation $\mathbf{B}=\nabla \times \mathbf{A}$, the null space is removed and thus $\mathbf{B}$ can be obtained correctly. However, $\mathbf{A}$ and the scalar potential $\Phi$ are of specific importance in the realm of quantum mechanics. For example, the Aharonov-Bohm effect [11] can be interpreted by $\mathbf{A}$; in addition, the electromagnetic effect can be incorporated into the Schrodinger equation in the form of $\mathbf{A}$ and $\Phi$ [12].

To obtain a unique solution of $\mathbf{A}$, the tree-cotree gauge condition [13] or the Coulomb gauge condition,

Manuscript received November 18, 2014; revised January 27, 2015; accepted March 14, 2015. Date of publication March 30, 2015; date of current version July 20, 2015. Corresponding author: S. Sun (e-mail: sunsheng@iee.org).

Color versions of one or more of the figures in this paper are available online at http://ieeexplore.iee.org.

Digital Object Identifier 10.1109/TMAG.2015.2417492 $\nabla \cdot \mathbf{A}=0$ [14], [15], should be implemented. In addition, the unguaged double curl matrix system becomes consistent when the current excitation is expressed by the curl of a source field, which makes the curl-curl equation is implicitly gauged by an iterative solver [16]. Nonetheless, the tree-cotree technique is not an optimal choice, because the resultant matrix is no longer highly sparse and the best tree graph, which leads to a fast convergence, is difficult to find. In addition, due to the existence of the divergence operator in the Coulomb gauge, nodal elements, instead of edge elements, are employed and thus $\mathbf{A}$ is decomposed into three components $\left(A_{x}, A_{y}\right.$, and $\left.A_{z}\right)$ [17], [18], which violated the vectorial nature of $\mathbf{A}$ and may bring about spurious modes.

In this paper, a vectorial solution to the double curl equation with a generalized Coulomb gauge is proposed to remedy the aforementioned drawbacks. In contrast to the traditional Coulomb gauged method, both the uniqueness and the vectorial nature of $\mathbf{A}$ are reserved in this paper. The generalized Coulomb gauge, which is the reduced form of the generalized Lorenz gauge [19] at static limit, is introduced to remove the null space of the curl operator and guarantees the uniqueness of $\mathbf{A}$. To retain the vectorial property of $\mathbf{A}$, it is expanded by the edge elements, and appropriate expansion of the gauge term is formulated based on the space mapping between the Whitney forms by mathematical operators (gradient, divergence, and curl operators) [20], [21], and the Hodge operators [22], [23]. The resultant matrix system has the same degrees of freedom (DoFs) with that of the original double curl equation. Furthermore, it is well conditioned, and thus can be solved by both direct and iterative solvers.

The remainder of this paper is organized as follows. The double curl equation with generalized Coulomb gauge is investigated and its finite-element discretization is derived in Section II. In Section III, the accuracy and effectiveness of the proposed method will be demonstrated by examining the matrix condition and comparing the results with that of the 


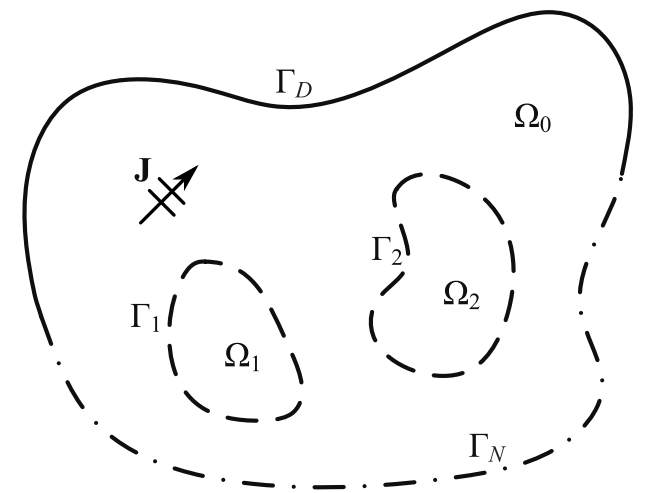

Fig. 1. General 3-D structure excited by an impressed current source $\mathbf{J}$.

original double curl equation. Finally, the conclusion is drawn in Section IV.

\section{FORMULATION}

\section{A. Governing Equation}

Consider a general 3-D boundary value problem, as shown in Fig. 1. Assume that the structure is inhomogeneously composed of three bodies, $\Omega_{0}, \Omega_{1}$, and $\Omega_{2}$, among which $\Omega_{0}$ is bounded by $\Gamma_{D}$ (solid line) and $\Gamma_{N}$ (dashed-dotted line); $\Omega_{1}$ and $\Omega_{2}$ are bounded by $\Gamma_{1}$ and $\Gamma_{2}$, respectively. In addition, the structure is excited by an impressed current source J. Thus, A satisfies the following double curl equation with generalized Coulomb gauge:

$$
\nabla \times \frac{1}{\mu} \nabla \times \mathbf{A}-\varepsilon \nabla \frac{1}{\alpha \varepsilon^{2} \mu} \nabla \cdot \varepsilon \mathbf{A}=\mathbf{J}
$$

which is the static case of the A-equation in [19], with $\varepsilon, \mu$, and $\alpha$ being the permittivity, permeability, and gauge factor, respectively. Meanwhile, the generalized Lorenz gauge

$$
\nabla \cdot \varepsilon \mathbf{A}=i \omega \alpha \mu \varepsilon^{2} \Phi
$$

is degenerated into the generalized Coulomb gauge

$$
\nabla \cdot \varepsilon \mathbf{A}=0 .
$$

It is worthwhile to point out that the generalized Coulomb gauge is not added to the double curl term intentionally, but embedded in (1) naturally, which is proved as follows. As $\mathbf{J}$ is solenoidal for magnetostatic cases, taking the divergence of both sides of (1) yields

$$
\nabla \cdot \varepsilon \nabla \frac{1}{\mu \varepsilon^{2}} \nabla \cdot \varepsilon \mathbf{A}=0
$$

which means A should be in the null space of resultant matrix system. It is well known that the matrix representation of the Laplace operator, $\nabla \cdot \varepsilon \nabla$, is positive definite. Hence, the null space of the operator $\nabla \cdot \varepsilon \nabla\left(1 / \mu \varepsilon^{2}\right) \nabla \cdot \varepsilon$ should also be the null space of the operator $\nabla \cdot \varepsilon$, which is indicated by (3). Since $\varepsilon$ may be inhomogeneous, (3) can be understood in a variational sense: testing (3) by a scalar potential $\varphi$ that is zero on the boundary, then integrating by part over the whole domain and employing the divergence theorem yield

$$
\int_{\Omega} \varepsilon \mathbf{A} \cdot \nabla \varphi d \Omega=0
$$

which claims that $\mathbf{A}$ is orthogonal to every irrotational field. In other words, $\mathbf{A}$ is solenoidal in a weak sense. When $\varepsilon$ is homogeneous, the generalized Coulomb gauge reduces to

$$
\nabla \cdot \mathbf{A}=0
$$

which is the common Coulomb gauge and states $\mathbf{A}$ is solenoidal in a rigorous sense. Furthermore, (1) can be rewritten as

$$
\nabla \times \frac{1}{\mu} \nabla \times \mathbf{A}-\nabla \frac{1}{\alpha \mu} \nabla \cdot \mathbf{A}=\mathbf{J}
$$

which is the traditional Coulomb gauged double curl equation. If the gauges are discarded, (1) and (7) will eventually be reduced into the so-called double curl equation

$$
\nabla \times \frac{1}{\mu} \nabla \times \mathbf{A}=\mathbf{J} .
$$

Based on the above analysis, one can see that the common Coulomb gauge is a special ( $\varepsilon$ is homogeneous) case of the generalized Coulomb gauge, and both gauges are embedded in (1) naturally. For simplicity but without loss of generality, the following homogeneous boundary conditions are assumed [18], [21]:

$$
\begin{aligned}
\hat{n} \times \mathbf{A}(\mathbf{r}) & =\mathbf{0}, & & \mathbf{r} \in \Gamma_{D} \\
\hat{n} \times\left(\frac{1}{\mu} \nabla \times \mathbf{A}(\mathbf{r})\right) & =\mathbf{0}, & & \mathbf{r} \in \Gamma_{N}
\end{aligned}
$$

where $\mathbf{r}$ denotes the observation position. Therefore, $\mathbf{A}$ is uniquely defined by (1), (3), (9), and (10). In addition, as indicated by (3), A should possess a rotation-like field profile.

\section{B. Finite-Element Discretization}

It is well known that edge elements, $\vec{\omega}(\mathbf{r})$, which belong to the Whitney-1 form, are suitable basis functions to represent $\mathbf{A}$

$$
\mathbf{A}=\sum_{n=1}^{N_{e}} a_{n} \vec{\omega}_{n}(\mathbf{r})
$$

where $N_{e}$ is the number of the edges in the discretized mesh, and $a_{n}$ is the corresponding unknown. However, as

$$
\nabla \cdot \vec{\omega}(\mathbf{r})=0
$$

the second term of (1) will vanish if edge elements are chosen, which makes (1) degenerate to ungauged double curl equation and thus vectorial expansion of $\mathbf{A}$ seems unavailable for (1) and (7). However, (12) does not mean that edge elements are solenoidal, since a constant gradient can be expanded in terms of edge elements [21]. This is the reason why a nonunique solution exists in (8) even though curl-conforming edge elements are used. To achieve the vectorial representation of $\mathbf{A}$ in (11), the trick is the divergence operation on edge elements can be bypassed by expanding the divergence term, as a whole, in the form of Whitney elements, since edge elements are actually not solenoidal.

According to differential forms theory [24], [25], the Hodge operator is a unique linear map on a semi-Riemmanian manifold from $p$-form to $(n-p)$-form, where $n$ is the 
dimension of the manifold ( $n=3$ for our discussion). By eyeballing both sides of (1), we find that the terms on the left-hand side (LHS) should lie in Whitney-2 form (flux space), which is consistent with its right-hand side. In other words, the $\nabla \times \mu^{-1} \nabla \times$ operator, which can be considered as a mixed (mathematical and Hodge) operator, projects a Whitney-1 form quantity into Whitney-2 form. Similarly, Whitney form transformation of the second term on the LHS can be understood in this way: the Whitney-1 form quantity is first projected into Whitney- 0 form, by a mixed operator $\left(\left(1 / \mu \varepsilon^{2}\right) \nabla \cdot \varepsilon\right)$, and then projected into Whitney-2 form, by another mixed operator $(\varepsilon \nabla)$. From this viewpoint, the Whitney forms can be mapped back and forth by the mathematical and Hodge operators.

Based on the above analysis, it is reasonable to arrive at

$$
\frac{1}{\mu \varepsilon^{2}} \nabla \cdot \varepsilon \mathbf{A}=\sum_{n=1}^{N_{n}} d_{n} \lambda_{n}(\mathbf{r})
$$

where $\lambda_{n}(\mathbf{r})$ is the nodal element, which belongs to Whitney- 0 form, $N_{n}$ is the number of the nodes in the discretized mesh, and $d_{n}$ is the corresponding unknown. To remove the additional DoF brought about by (13), testing both sides of (13) with $\lambda_{m}(\mathbf{r})$ yields

$$
\sum_{n=1}^{N_{e}}\left\langle\lambda_{m}, \frac{1}{\mu \varepsilon^{2}} \nabla \cdot \varepsilon \vec{\omega}_{n}\right\rangle a_{n}=\sum_{n=1}^{N_{n}}\left\langle\lambda_{m}, \lambda_{n}\right\rangle d_{n} .
$$

Thus, the unknown vector $\{d\}$ can be written in the form of the unknown vector $\{a\}$, i.e.

$$
\{d\}=\left[G_{N N}\right]^{-1}\left[G_{N E}\right]\{a\}
$$

where $\left[G_{N N}\right]$ and $\left[G_{N E}\right]$ are $N_{n} \times N_{n}$ matrix and $N_{n} \times N_{e}$ matrices, respectively. They are defined as

$$
\begin{aligned}
{\left[G_{N N}\right]_{m n} } & =\int_{\Omega} \lambda_{m} \cdot \lambda_{n} d \Omega \\
{\left[G_{N E}\right]_{m n} } & =\int_{\Gamma} \frac{1}{\mu \varepsilon} \lambda_{m} \vec{\omega}_{n} \cdot \hat{n} d \Gamma-\int_{\Omega} \frac{1}{\mu \varepsilon} \nabla \lambda_{m} \cdot \vec{\omega}_{n} d \Omega .
\end{aligned}
$$

Finally, testing both sides of (1) with $\vec{\omega}_{m}(\mathbf{r})$ and applying (9), (10), (11), and (15) result in

$$
\left\{\left[K_{1}\right]-\left[K_{2}\right]\left[G_{N N}\right]^{-1}\left[G_{N E}\right]\right\}\{a\}=\{b\}
$$

where [ $\left.K_{1}\right]$ is an $N_{e} \times N_{e}$ matrix, [ $\left.K_{2}\right]$ is an $N_{e} \times N_{n}$ matrix, and $\{b\}$ is an $N_{e} \times 1$ vector, and they are defined as

$$
\begin{aligned}
{\left[K_{1}\right]_{m n} } & =\int_{\Omega}\left[\mu^{-1}\left(\nabla \times \vec{\omega}_{m}\right) \cdot\left(\nabla \times \vec{\omega}_{n}\right)\right] d \Omega \\
{\left[K_{2}\right]_{m n} } & =\int_{\Omega} \vec{\omega}_{m} \cdot \nabla \lambda_{n} d \Omega \\
\{b\}_{m} & =\int_{\Omega} \vec{\omega}_{m} \cdot \mathbf{J} d \Omega .
\end{aligned}
$$

From the above derivation, a clear clue of how to discretize (1) using FEM is demonstrated. However, it is not that obvious in (7), which might be the reason why nodal discretization is employed. Noting that (6) is actually the homogeneous case of (3) and (7) can be rewritten as

$$
\nabla \times \frac{1}{\mu} \nabla \times \mathbf{A}-\varepsilon_{0} \nabla \frac{1}{\alpha \varepsilon_{0}^{2} \mu} \nabla \cdot \varepsilon_{0} \mathbf{A}=\mathbf{J}
$$

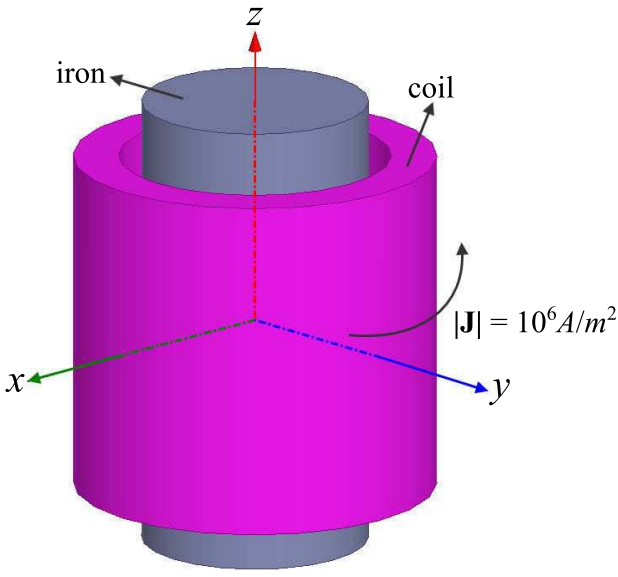

(a)

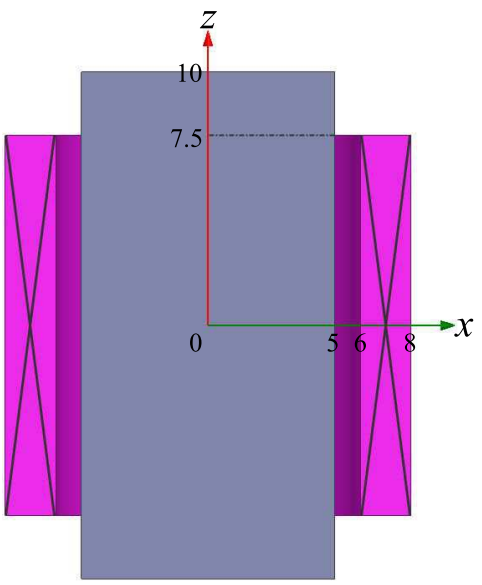

(b)

Fig. 2. Iron cylinder with $\mu_{r}=1000$ is immersed in the field of a cylindrical coil. (a) 3-D view. (b) Sectional view (unit: centimeter)

where the clue emerges obviously. It is worthy of mentioning that (1) and (22) are static and static-homogeneous cases of (2). Hence, the physically meaningful nature of the generalized Lorenz gauge is inherited and at the same time, magnetostatic potential equations and dynamic potential equations are unified under the same framework based on the generalized Lorenz gauge and generalized Coulomb gauge. It can be observed that the solutions of (1) and (22) satisfy (8) automatically, as $\mathbf{A}$ is gauged to be solenoidal in a weak/rigorous sense. Basically, the solution of (1) is different from (22), since different gauges are applied and different irrotational components of $\mathbf{A}$, which are brought about by numerical errors, exist in the solutions. However, these components will be removed completely when $\mathbf{B}$ is solved by $\mathbf{B}=\nabla \times \mathbf{A}$. Finally, the same solution of $\mathbf{B}$ will be achieved by (1), (8), and (22).

Since the second term on the LHS of (1) and (22) involves matrix inverse and multiplication, it may cost additional computation time and memory. Because of sparse approximate inverse (SAI) [26] technique, $\left[G_{N N}\right]^{-1}$ can be approximated by the SAI of $\left[G_{N N}\right]$, instead of the exact inverse, without much loss of accuracy. This approximation has been investigated in [24] and [28]. The point is that $\mathbf{A}$, which, required by (3), is weakly solenoidal, will not be affected 


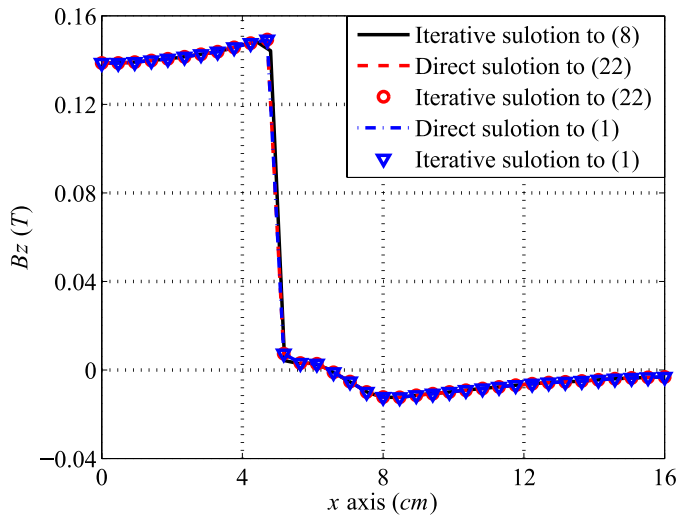

(a)

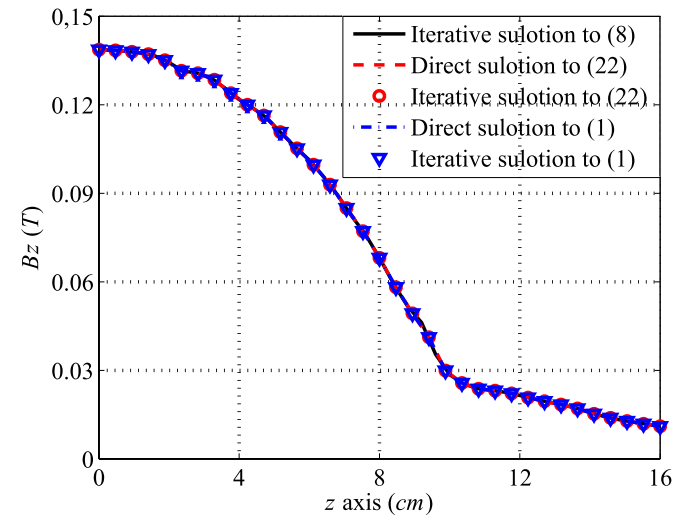

(b)

Fig. 3. (a) $B_{z}$ along $x$-axis $(\alpha=1$ and $\beta=0.6)$. (b) $B_{z}$ along $z$-axis $(\alpha=1$ and $\beta=0.6)$.

TABLE I

Statistics of Resultant Matrices With DIFFERENT FORMULATION

\begin{tabular}{|c|c|c|}
\hline Formulation & Condition number & Iterative steps \\
\hline$(8)$ & -1 & 197 \\
\hline$(1)^{2}$ & $1.9732 \times 10^{7}$ & 2804 \\
\hline$(22)$ & $3.8417 \times 10^{6}$ & 869 \\
\hline
\end{tabular}

${ }^{1}$ the matrix system of $(8)$ is singular;

2 assume $\varepsilon=10 \varepsilon_{0}$ in the iron and $\varepsilon=\varepsilon_{0}$ everywhere else.

by the simplification. In the numerical implementation, the quality and cost of computing $\left[G_{N N}\right]^{-1}$ is controlled by $\beta(\beta \in[0,1])$. In general, lower values of $\beta$ lead to more computation, more fill-in, but better approximation of the real inverse. If one keeps reducing $\beta$, SAI will eventually compute the exact inverse.

\section{NUMERICAL VERIFICATION}

Here, an example of cylinder iron in the field of a cylindrical coil [28], as shown in Fig. 2, is presented to verify the proposed solution for magnetostatic problems. As the structure is axial symmetrical, we can discretize the portion lying in the first quadrant instead of the whole domain.

As the null space of the curl operator is removed, direct solvers can be applied to (1) and (22). In addition, iterative

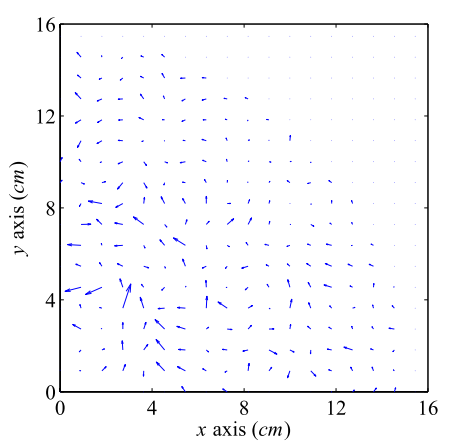

(a)

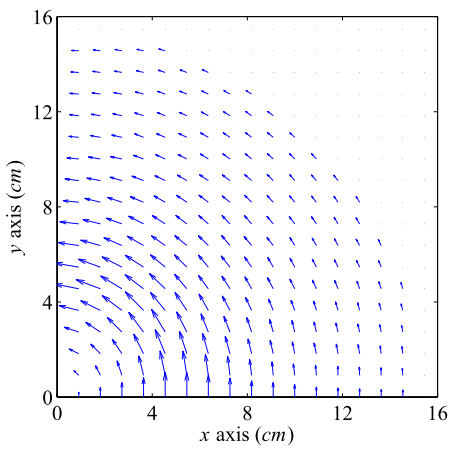

(c)

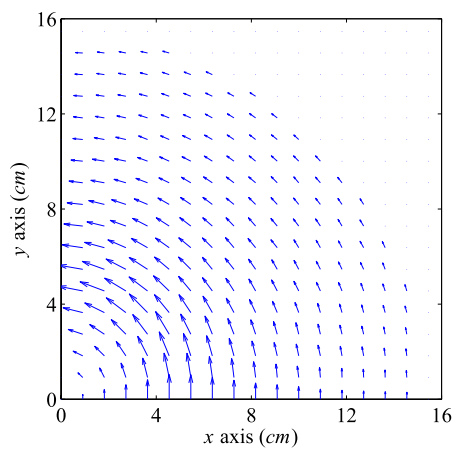

(e)

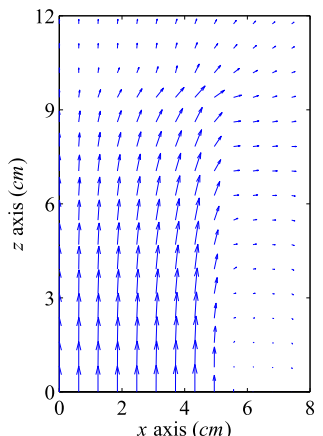

(b)

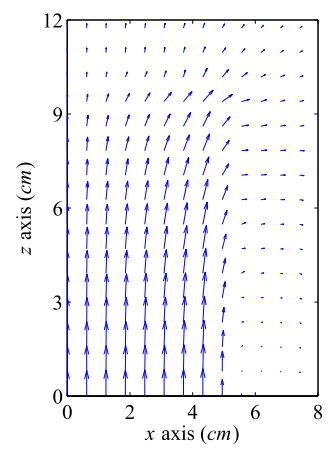

(d)

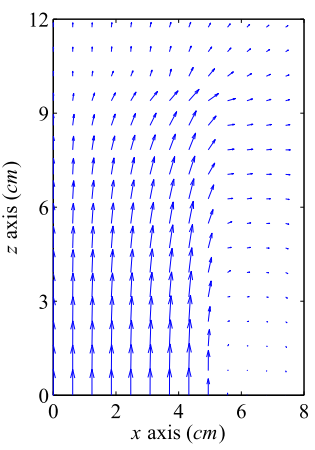

(f)
Fig. 4. Vector plots of $\mathbf{A}$ in $x y$ plane, obtained by (a) (8), (c) (1), and (e) (22). Vector plots of $\mathbf{A}$ and $\mathbf{B}$, where (a) and (b) obtained by (8); (c) and (d) obtained by (1); (e) and (f) obtained by (22), respectively.

solvers are good candidates for solving the matrix system, as the condition number of the matrix is well controlled. Fig. 3 presents $B_{z}$ values, obtained by the proposed method using both direct and iterative solvers, along the axes. The result obtained by solving (8) is added for reference. It is obvious that these results are in overlap agreement with each other, which demonstrates the accuracy of the proposed method and supports the observation in Section II. In addition, the corresponding condition numbers and generalized minimal residual (GMRES) [29] iterative steps used to achieve $10^{-6}$ accuracy of the solution are listed in Table I. Although the singular system (8) converges faster due to the compatibility of the matrix system [16], its solution is not unique. Obviously, the inhomogeneity of $\varepsilon$ worsens the matrix condition and thus more iterative steps are needed for (1). Hence, (22) is preferred for magnetostatic applications.

In addition, $\mathbf{A}$ vector and $\mathbf{B}$ vector on the $x y$ plane and $x z$ plane, respectively, are shown in Fig. 4. Apparently, 


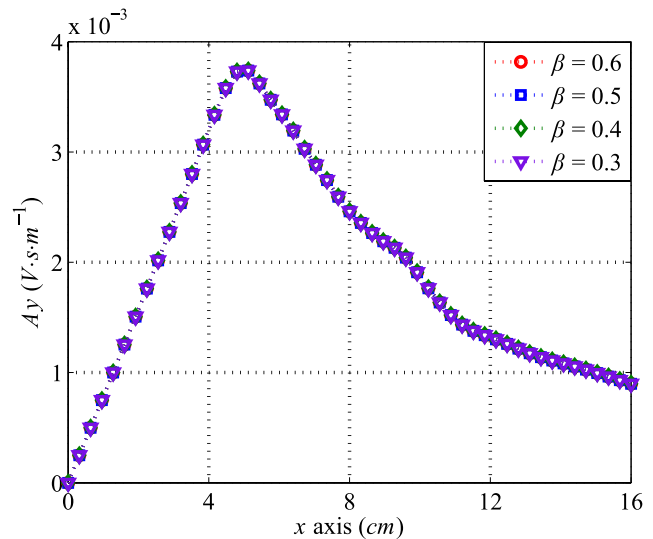

(a)

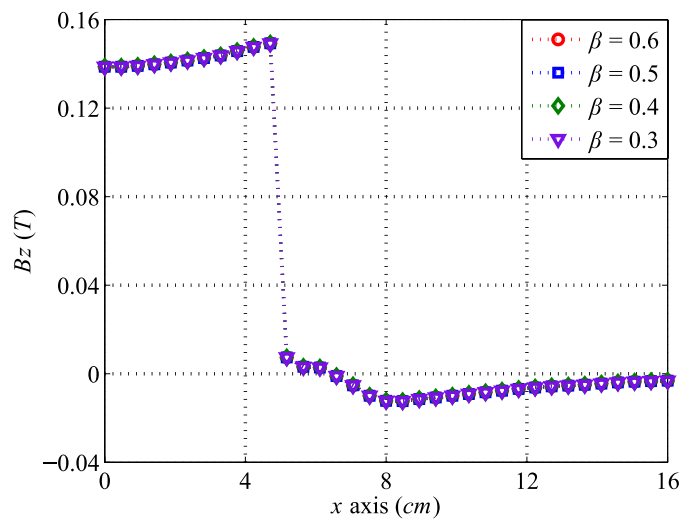

(b)

Fig. 5. (a) $A_{y}$ and (b) $B_{z}$ obtained by solving (22) with different $\beta$ values.

TABLE II

Statistics of Resultant Matrix of (22) With DifFerent $\beta$

\begin{tabular}{|c|c|c|c|}
\hline$\beta$ & Sparsity ${ }^{1}$ & Condition number & Iterative steps ${ }^{2}$ \\
\hline 0.6 & $1.3529 \times 10^{-2}$ & $3.8417 \times 10^{6}$ & 869 \\
\hline 0.5 & $1.3558 \times 10^{-2}$ & $3.9867 \times 10^{6}$ & 888 \\
\hline 0.4 & $2.2700 \times 10^{-2}$ & $5.0017 \times 10^{6}$ & 989 \\
\hline 0.3 & $4.8542 \times 10^{-2}$ & $5.9337 \times 10^{6}$ & 1497 \\
\hline
\end{tabular}

B profiles obtained by (1), (8), and (22) are almost the same, which make sense as the null space of $\mathbf{A}$ is eliminated by the curl operation, $\mathbf{B}=\nabla \times \mathbf{A}$. However, the nonuniqueness exists in (8), which makes iterative solvers converge to different solutions with different initial guesses, while this is not the case for (1) and (22). In addition, the gauge effect plays an important role for A. As shown in Fig. 4, A profiles obtained by (1) and (22) are smooth and flow in a rotational sense, as required by (3) and (6), while that obtained by (8) looks messy.

Furthermore, as $\left[G_{N N}\right]^{-1}$ is controlled by $\beta$, the matrix condition should be influenced by $\beta$. Table II summarizes the variation of sparsity and condition number of the resultant matrix, $\left[K_{1}\right]-\left[K_{2}\right]\left[G_{N N}\right]^{-1}\left[G_{N E}\right]$, as well as GMRES [29] iterative steps used to achieve $10^{-6}$ accuracy of the solution. It is worth noting that the sparsity of $\left[G_{N N}\right]^{-1}$, obtained by SAI, is $<7.7660 \times 10^{-4}$, which means $\left[G_{N N}\right]^{-1}$ can be approximated by a near-diagonal matrix without perceptible loss of accuracy, as presented in Fig. 5. Hence, the sparse merit of the final matrix obtained by FEM is greatly retained. In addition, if iterative solvers are used, the inverse of $\left[G_{N N}\right]$ is not necessary, because the solution can be found based on the inner and outer iterative procedures.

\section{CONCLUSION}

The double curl equation with generalized Coulomb gauge for magnetostatic problems has been solved by expanding A with tangentially continuous vector elements. The null space of the curl operator is removed by the generalized Coulomb gauge, which regulates $\mathbf{A}$ to be solenoidal in a weak sense, and thus the uniqueness of $\mathbf{A}$ is guaranteed. The mapping between Whitney forms by mixed operators is investigated, which inspires an appropriate approximation of the gauge term. Approximating $\left[G_{N N}\right]^{-1}$ by the SAI of $\left[G_{N N}\right]$, the resultant matrix retains the sparse property of FEM, and is well conditioned at the same time. Hence, both direct and iterative solvers can be applied. The numerical results are in perfect agreement with the reference and good consistency with the physics.

\section{ACKNOWLEDGMENT}

This work was supported in part by the Research Grants Council, Hong Kong, under Grant GRF 716112, Grant 716713, and Grant 17207114, and in part by the University Grants Council, Hong Kong, under Contract AoE/P-04/08, Contract 201211159076, Contract 201209160031, and Contract 201311159188. The work of W. C. Chew was supported by the National Science Foundation CCF under Award 1218552 and SRC Task 2347.001.

\section{REFERENCES}

[1] B. T. Dalslet, M. Donolato, and M. F. Hansen, "Planar Hall effect sensor with magnetostatic compensation layer," Sens. Actuators A, Phys., vol. 174, pp. 1-8, Feb. 2012.

[2] R. Ravaud, G. Lemarquand, S. Babic, V. Lemarquand, and C. Akyel, "Cylindrical magnets and coils: Fields, forces, and inductances," IEEE Trans. Magn., vol. 46, no. 9, pp. 3585-3590, Sep. 2010.

[3] T. Tadic and B. G. Fallone, "Design and optimization of a novel bored biplanar permanent-magnet assembly for hybrid magnetic resonance imaging systems," IEEE Trans. Magn., vol. 46, no. 12, pp. 4052-4058, Dec. 2010.

[4] F. Kikuchi, "Mixed formulations for finite element analysis of magnetostatic and electrostatic problems," Jpn. J. Appl. Math., vol. 6, no. 2, pp. 209-221, Jun. 1989.

[5] H. Kanayama, H. Motoyama, K. Endo, and F. Kikuchi, "Threedimensional magnetostatic analysis using Nedelec's elements," IEEE Trans. Magn., vol. 26, no. 2, pp. 682-685, Mar. 1990.

[6] K. Preis, I. Bardi, O. Biro, C. Magele, G. Vrisk, and K. R. Richter, "Different finite element formulations of 3D magnetostatic fields," IEEE Trans. Magn., vol. 28, no. 2, pp. 1056-1059, Mar. 1992.

[7] P. Alotto and I. Perugia, "A field-based finite element method for magnetostatics derived from an error minimization approach," Int. J. Numer. Methods Eng., vol. 49, no. 4, pp. 573-598, Oct. 2000

[8] A. Bermúdez, R. Rodríguez, and P. Salgado, "A finite element method for the magnetostatic problem in terms of scalar potentials," SIAM J. Numer. Anal., vol. 46, no. 3, pp. 1338-1363, 2008.

[9] Getting Started With Maxwell, ANSYS, Inc., Canonsburg, PA, USA, 2010. 
[10] D. Meeker. (2010). User's Manual of Finite Element Method Magnetics, Version 4.2

[11] Y. Aharonov and D. Bohm, "Significance of electromagnetic potentials in the quantum theory," Phys. Rev., vol. 115, p. 485, Aug. 1959.

[12] M. Fox, Quantum Optics: An Introduction, vol. 15. London, U.K.: Oxford Univ. Press, 2006

[13] J. B. Manges and Z. J. Cendes, "A generalized tree-cotree gauge for magnetic field computation," IEEE Trans. Magn., vol. 31, no. 3, pp. 1342-1347, May 1995.

[14] O. Biro and K. Preis, "On the use of the magnetic vector potential in the finite-element analysis of three-dimensional eddy currents," IEEE Trans. Magn., vol. 25, no. 4, pp. 3145-3159, Jul. 1989.

[15] K. Preis et al., "Numerical analysis of 3D magnetostatic fields," IEEE Trans. Magn., vol. 27, no. 5, pp. 3798-3803, Sep. 1991.

[16] Z. Ren, "Influence of the RHS on the convergence behaviour of the curl-curl equation," IEEE Trans. Magn., vol. 32, no. 3, pp. 655-658, May 1996.

[17] O. Biro, K. Preis, and K. R. Richter, "On the use of the magnetic vector potential in the nodal and edge finite element analysis of 3D magnetostatic problems," IEEE Trans. Magn., vol. 32, no. 3, pp. 651-654, May 1996.

[18] J.-M. Jin, The Finite Element Method in Electromagnetics, 2nd ed. New York, NY, USA: Wiley, 2002.

[19] W. C. Chew, "Vector potential electromagnetics with generalized gauge for inhomogeneous media: Formulation," Prog. Electromagn. Res. vol. 149, pp. 69-84, 2014.
[20] A. Bossavit, "Whitney forms: A class of finite elements for threedimensional computations in electromagnetism," IEE Proc. A, Phys. Sci., Meas. Instrum., Manage. Edu.-Rev., vol. 135, no. 8, pp. 493-500, Nov. 1988.

[21] Y. Zhu and A. C. Cangellaris, Eds., Multigrid Finite Element Methods for Electromagnetic Field Modeling. New York, NY, USA: Wiley, 2006.

[22] B. He, "Compatible discretizations for Maxwell equations," Ph.D. dissertation, Dept. Elect. Compt. Eng., Ohio State Univ., Columbus, OH, USA, 2006.

[23] Q. I. Dai, W. C. Chew, and L. J. Jiang, "Differential forms inspired discretization for finite element analysis of inhomogeneous waveguides (invited paper)," Prog. Electromagn. Res., vol. 143, pp. 745-760, 2013.

[24] G. A. Deschamps, "Electromagnetics and differential forms," Proc. IEEE, vol. 69, no. 6, pp. 676-696, Jun. 1981.

[25] I. V. Lindell, Differential Forms in Electromagnetics. New York, NY, USA: Wiley, 2004

[26] M. J. Grote and T. Huckle, "Parallel preconditioning with sparse approximate inverses," SIAM J. Sci. Comput., vol. 18, no. 3 , pp. 838-853, May 1997.

[27] D. A. White and J. M. Koning, "Computing solenoidal eigenmodes of the vector Helmholtz equation: A novel approach," IEEE Trans. Magn., vol. 38 , no. 5, pp. 3420-3425, Sep. 2002.

[28] C. Magele, H. Stogner, and K. Preis, "Comparison of different finite element formulations for 3D magnetostatic problems," IEEE Trans. Magn., vol. 24, no. 1, pp. 31-34, Jan. 1988.

[29] Y. Saad and M. H. Schultz, "GMRES: A generalized minimal residual algorithm for solving nonsymmetric linear systems," SIAM J. Sci. Statist. Comput., vol. 7, no. 3, pp. 856-869, 1986. 